\title{
G-CSF related hemophagocytosis in a case with lymhphoma
}

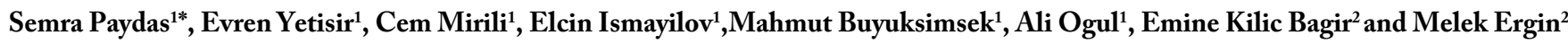 \\ ${ }^{1}$ Faculty of Medicine Department of Medical Oncology, Cukurova University, Adana /Turkey \\ ${ }^{2}$ Faculty of Medicine, Department of Pathology, Adana /Turkey
}

\begin{abstract}
Hemophagocytic syndrome (HS) is relatively rare condition, generally is seen in children and is characterized by fever, hepatosplenomegaly and cytopenias with some serologic findings. Several inflammatory cytokines including interferon gamma, interleukin-2 and tumor necrosis factor are responsible from this clinical table. This syndrome may be seen as primary- congenital immune disorder or it may develop secondary to infection, autoimmune diseases and malignancies [1]. Hemophagocytosis is characterized by histiocytic phagocytosis of erythrocytes, leukocytes, platelets or precursor of these cells and may be seen in various clinical conditions including infections, inflammations, drugs and toxins. Here we described a 50-years-old man with lymphoma developing hemophagocytosis associated with G-CSF using and response to steroid.
\end{abstract}

\section{Case report}

Fifty years old man admitted to the hospital with epigastric pain. Endoscopic biopsy showed non-Hodgkin lymphoma-diffuse large B cell lymphoma with high Ki-67 score (90\%). PET/CT showed diffuse gastric infiltration, disseminated abdominal lymph nodes with 3-5 $\mathrm{cm}$ diameters. Bone marrow biopsy was negative for lymphoma infiltration. Patient was treated by prephase therapy to prevent tumor lysis syndrome between May 2 and 8, 2017 due to high tumor burden and high proliferative index. After this therapy DA-EPOCH-R was given between May 10 and 14, 2017 and G-CSF 45MU daily was prescribed on May 16, 2017 and was stopped on May 22, 2017 when WBC was $8.5 \times 10^{9} / \mathrm{L}$. However on May 18, 2017 acute abdominal pain developed, abdominal CT showed perforation and abdominal surgery was done immediately. Surgery was uncomplicated but sudden drop in hemoglobin and hematocrit levels had been observed in surgery clinic despite no bleeding intra or postoperative periods. Six units of erythrocyte suspensions had been given to the patient between 20 and 25 May, 2017. Patient was consulted by us at surgical department due to severe anemia on May 26, 2017; hemoglobin was 4.8g/dl, Hct was $14 \%$, WBC count was $46 \times 10^{9} / \mathrm{L}$, platelet count was $280 \times 10^{9} / \mathrm{L}$, LDH was 697 IU. Daily WBC, hemoglobin, hematocrit, platelet count changes have been shown in Figures 1a, 1b, 1c. There was aniso-poikilocytosis, polychromatophilia, normoblasts, left shift in WBC series in peripheral blood smear. Coombs test was found to be negative, bilirubin levels were within normal limits. Due to abnormal peripheral blood findings bone marrow aspiration and biopsy were done. Marrow aspiration was hypercellular and there was macrophages showing hemphagocytosis and bone marrow biopsy showed hemophagocytosis. Prednisolone $100 \mathrm{mg}$ daily was prescribed, drops in hemoglobin levels decreased; no transfusion was given after steroid injections and last hemoglobin level is $7.2 \mathrm{~g} / \mathrm{dl}$.

\section{Discussion}

HS is a complex clinical presentation causing diagnostic problems in daily practice. There are many etiologic factors in the development of HS and variable etiologic factors and clinical presentations may be challenging for clinicians. For this reason diagnosis may be late and morbidity may increase. In fact, hemophagocytosis is relatively more commonly seen in daily practice and if patient is not followed carefully it may be overlooked and hemophagocytosis may not be considered. In our patient at the beginning the cause of sudden decreases in hemoglobin and hematocrit levels had not been evaluated and blood transfusion had been done due to low hemoglobin levels in department of surgery. When we look the patient at the surgery clinic, there was no evidence of hemolysis and also there was no evidence of internal/

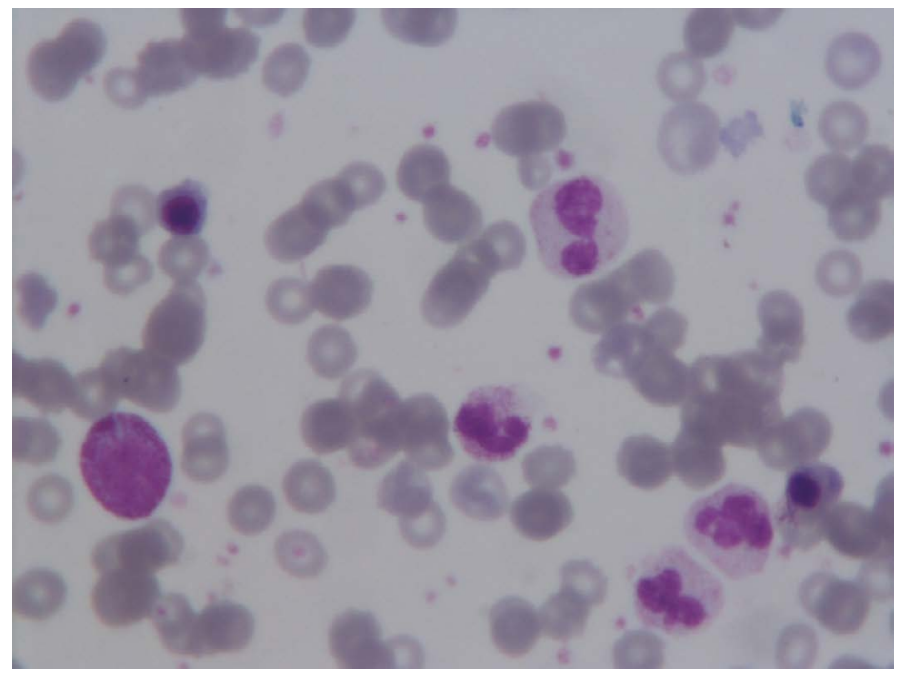

Figure 1a. Peripheral blood smear on May 26 showing left shift, leukocytosis and normoblasts.

Correspondence to: Semra Paydas, Faculty of Medicine Department of Medical Oncology, Cukurova University, Adana /Turkey, E-mail: sepay@cu.edu.tr

Received: December 05, 2017; Accepted: January 19, 2018; Published: January 22,2018 


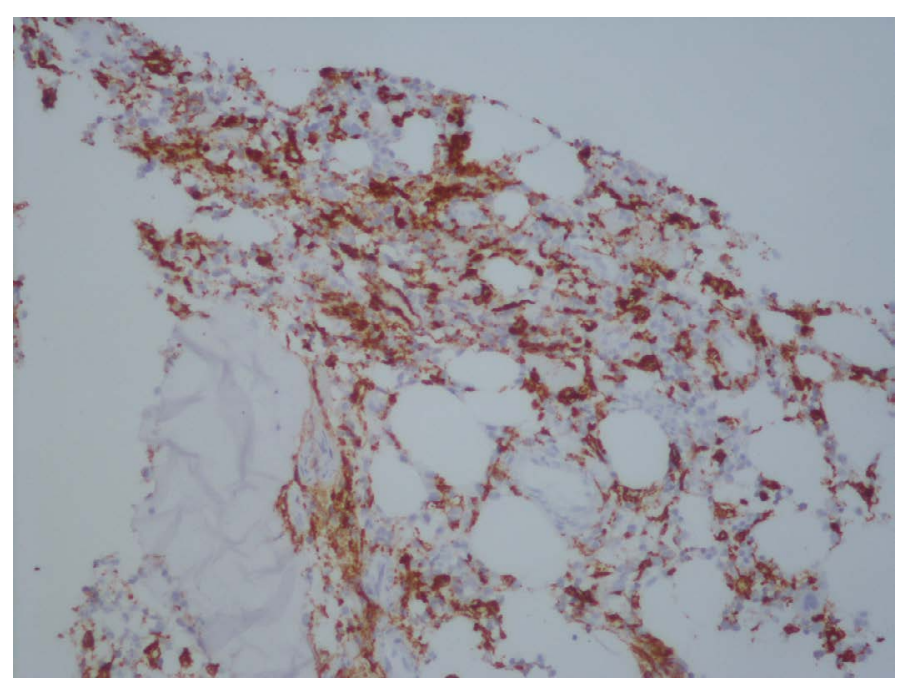

Figure 1b. Bone marrow biopsy showing CD163 positivity.

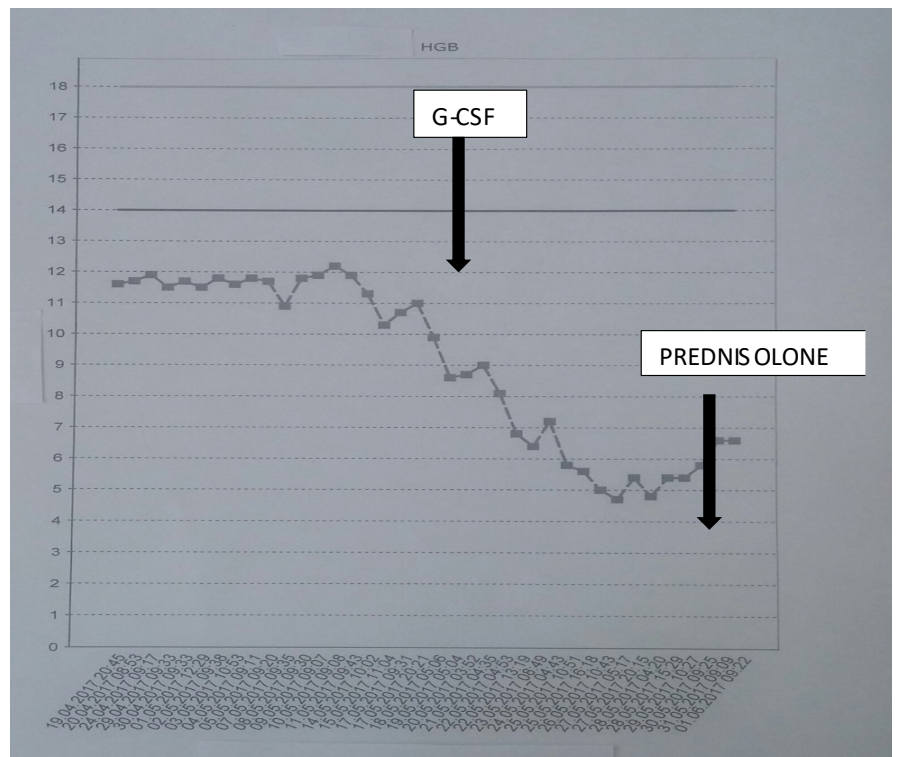

Figure 1c. Hemoglobin levels during clinical outcome, G-CSF using, prednisolone prescription.

external bleeding defining severe anemia. Peripheral blood smear suggested severe bone marrow irritation and bone marrow aspiration sample showed hemophagocytosis. When we reviewed the patient's clinical outcome and medications we think that G-CSF could be an etiologic factor for hemophagocytosis.

G-CSF is very frequently used cytokine in hematology/oncology clinics in neutropenic patients or neutropenia prophylaxis in high risk patients. The aim of our G-CSF use was prophylaxis, because $\mathrm{DA}-\mathrm{EPOCH}$ is regimen with high risk for neutropenia. G-CSF has many side effects including bone pain, pulmonary toxicity, neutrophilic dermatoses, splenic rupture, microemboli and others [26]. Hemophagocytosis associated with G-CSF using has been reported in three cases. First case has been reported 20 years ago, this case had juvenile rheumatoid arthritis and accompanying HS had been treated by cyclosporine and also she received $1 \mu \mathrm{g} / \mathrm{kg}$ of G-CSF for neutropenia. However G-CSF dosage had been increased to $5 \mu \mathrm{g} / \mathrm{kg}$ due to sufficient improvement in neutropenia. Relapse of HS had been detected at this dose of G-CSF. Authors suggested that higher dose of cytokine caused HS and G-CSF must be used in lower doses in these cases [7]. Second case had MDS and accompanying HS and had been treated by G-CSF plus GM-CSF due to neutropenia. Splenomegaly and severe thrombocytopenia developed with these cytokines and this table recurred with re-challenge of these cytokines and authors proposed that myeloid growth factors may be detrimental in patients with MDS-associated hemophagocytosis [8]. Last case has been reported 10 years ago, he was MDS and he had been treated by long acting G-CSF (pegfilgrastim). Histiocytic hemophagocytosis and blast increase developed with long acting cytokine and clinical table regressed after discontinuation of G-CSF [1]. Authors postulated that long acting G-CSF reacted with G-CSF receptors on monocytes, provided continuous stimulation with pharmacologic rather than physiologic concentrations of growth factor and direct stimulation of monocytes caused the proliferation of histiocytes and activated hemophagocytosis [9].

It is very well known that G-CSF has a key role in macrophage and antigen presenting cell activation. HS is related with T cell dysregulation and broad spectrum inflammatory cytokines are responsible from HS. G-CSF is a broad spectrum cytokine. Among these 3 cases, G-CSF using caused exacerbation of preexisting HS in first two cases. In last case long acting G-CSF caused de-novo hemophagocytosis. In our case anemia was the dominant finding of HS and steroid response was good.

In conclusion G-CSF may cause HS and must be thought in cases treated by this cytokine in differential diagnosis if develops unexpected cytopenias and/or HS suggesting signs and symptoms. On the other hand pegylated form of this cytokine may be more potent for these entities.

\section{Conflict of interest}

Authors declare that there is no conflict of interest

\section{References}

1. Brisse E, Matthys P, Wouters CH (2016) Understanding the spectrum of haemophagocytic lymphohistiocytosis: update on diagnostic challenges and therapeutic options. Br J Haematol 174: 175-187.

2. Nuamah NM, Goker H, Kilic YA, Damoura H, Cakmak A (2006) Spontaneous splenic rupture in a healthy allogeneic donor of peripheral-blood stem cell following the administration of granulocyte colony-stimulating factor (g-csf). A case report and review of the literatüre. Haematologica 91: 26-28.

3. Yoshida I, Matsuo K, Teshima T, Hashimoto D, Tanimoto Y, et al. (2006) Transient respiratory disturbance by granulocyte-colony-stimulating factor administration in healthy donors of allogeneic peripheral blood progenitor cell transplantation. Transfusion 46: 186-192.

4. PaydaÅŸ S, Sahin B, Seyrek E, Soylu M, Gonlusen G, et al. (1993) Sweet's syndrome associated with G-CSF. Br J Haematol 85: 191-192. [Crossref]

5. Akizuki S, Mizorogi F, Inoue T, Sudo K, Ohnishi A (2000) Pharmacokinetics and adverse events following 5-day administration of lenograstim, a recombinant human granulocyte colony-stimulating factor, in healthy subjects. Bone Marrow Transp 26: 939-946.

6. Martino M, Massara E, Irrera G, Messina G, Barreca G, et al. (2012) Pulmonary miçoembolism in a healthy donor following G-CSF administration for mobilization of hemopoietic progentior cells. Bone Marrow Transp 47: 308-310.

7. Quesnel B, Catteau B, Aznar V, Bauters F, Fenaux P (1997) Successful treatment of juvenile rheumatoid arthritis-associated haemophagocytic syndrome by cyclosporin A with a transient exacerbation by conventional dose G-CSF. Br J Haematol 97: 508-510.

8. Wang S, Degar BA, Zieske A, Shafi NQ, Rose MG (2004) Hemophagocytosis exacerbated by G-CSF/GM-CSF treatment in a patient with myelodysplasia. Am J Hematol 77: 391-396.

9. Glasser L, LeGolvan M, Horwitz HM (2007) Florid histiocytic hemophagocytosi following therapy with long acting G-CSF (pegfilgrastim). Am J Hematol 82: 753-757.

Copyright: (C2018 Paydas S. This is an open-access article distributed under the terms of the Creative Commons Attribution License, which permits unrestricted use, distribution, and reproduction in any medium, provided the original author and source are credited. 